Forum $2022 \cdot 37: 123-128$ Online publiziert: 4. März 2022

() The Author(s), under exclusive licence to Springer Medizin Verlag GmbH, ein Teil von Springer Nature 2022

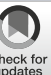

https://doi.org/10.1007/s12312-022-01055-x

\section{Tabakkonsum und Behandlung von Tabakabhängigkeit}

\author{
Wirksame Strategien und aktuelle Entwicklungen
}

\author{
Katja Leuteritz ${ }^{1}$ Veit Zebralla ${ }^{2} \cdot$ Hubert Wirtz $^{3}$ \\ 'Abteilung für Medizinische Psychologie und Medizinische Soziologie, Universitätsklinikum Leipzig, \\ Leipzig, Deutschland \\ ${ }^{2}$ Klinik und Poliklinik für Hals-, Nasen- und Ohrenheilkunde, Universitätsklinikum Leipzig, Leipzig, \\ Deutschland \\ ${ }^{3}$ Klinik und Poliklinik für Pneumologie, Universitätsklinikum Leipzig, Leipzig, Deutschland
}

Zusammenfassung

\section{In diesem Beitrag}

- Tabakentwöhnung als adjuvante Therapie bei Tumorerkrankungen

- Diagnostik und Behandlung von schädlichem und abhängigem Tabakkonsum

- Gesundheitspolitische Rahmenbedingungen für Tabakentwöhnung in Deutschland
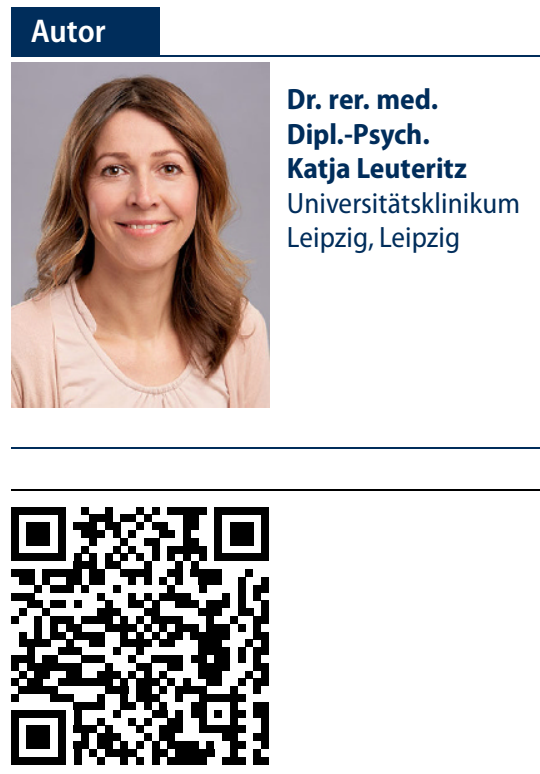

QR-Code scannen \& Beitrag online lesen

Jedes Jahr sterben in Deutschland rund 127.000 Menschen an den Folgen des Rauchens. Darunter befinden sich 85.000 Menschen mit einer tabakassoziierten Krebserkrankung. Dennoch raucht in Deutschland immer noch etwa ein Viertel der erwachsenen Bevölkerung, oftmals auch dann noch, wenn eine tabakassoziierte Erkrankung auftritt. Viele Rauchende schaffen die Abstinenz nicht ohne Unterstützung. Dieser Beitrag gibt einen Überblick über wirksame Strategien zur Tabakentwöhnung und beschreibt das Potenzial der Tabakentwöhnung im Rahmen der adjuvanten Therapie bei Tumorerkrankungen. Darüber hinaus werden gesundheitspolitische Herausforderungen im Rahmen der Versorgung zur Tabakentwöhnung aufgezeigt. Eine Vielzahl von psychologischen und pharmakologischen Interventionsmethoden zur Tabakentwöhnung ist wirksam. Rauchenden Patienten und Patientinnen mit tabakassoziierten Erkrankungen soll eine Tabakentwöhnungstherapie angeboten werden. Tabakentwöhnung ist als adjuvante Therapie bei Tumorerkrankungen von großer Bedeutung. Sie verursacht vergleichsweise geringe Kosten und bringt erhebliche Vorteile für die Krankheitsgenesung und Lebensqualität der Betroffenen. Allerdings wird sie in Deutschland nur als wenig regulierte Präventionsmaßnahme angeboten. Zur Verbesserung der Lebensqualität einer Vielzahl von Menschen mit tabakassoziierten Erkrankungen und aus gesundheitsökonomischen Gründen ist es dringend erforderlich, Tabakentwöhnung als flächendeckenden Routineprozess in der medizinischen Versorgung zu etablieren und zu finanzieren.

\section{Schlüsselwörter}

Nikotin · Rauchentwöhnung · Krebs · Prävention · Interventionen
Tabakrauch enthält über 250 giftige und 90 krebserzeugende oder möglichweise krebserzeugende Substanzen. Rauchen schädigt nahezu jedes Organ des Körpers und ist der größte vermeidbare Risikofaktor für chronische nichtübertragbare Krankheiten. Jedes Jahr sterben in Deutschland rund 127.000 Menschen an den Folgen des Rauchens [1]. Dies entspricht bundesweit jedem 7. Todesfall. Etwa jede 5. Krebsneuerkrankung in Deutschland ist eine Folge des Rauchens jährlich sind dies etwa 85.000 Krebsfälle, die sich vermeiden ließen [1]. Rund
80-90\% aller Lungenkrebsfälle lassen sich in Deutschland auf Tabakkonsum zurückführen [2]. Rauchende haben zudem im Vergleich zu Nichtrauchenden ein mehr als doppelt so hohes Risiko für HerzKreislauf-Erkrankungen und ein doppelt so hohes Risiko für Schlaganfälle sowie einen schweren COVID-19-Verlauf [3, 4]. Auch nichttägliches Gelegenheitsrauchen ist mit einem Verlust an Lebensjahren verbunden [5].

Dennoch raucht in Deutschland etwa jeder vierte Erwachsene Tabak, wobei Männer häufiger $(27,1 \%)$ als Frauen 
$(19,1 \%)$ rauchen. Mit zunehmendem Lebensalter steigen die Häufigkeit und die Menge des Rauchens in Deutschland an. Das Durchschnittsalter für den Beginn des täglichen Tabakkonsums liegt in Deutschland bei 16 Jahren [1, 6]. Etwa die Hälfte der aktuell rauchenden Personen erfüllt die Kriterien für eine Tabakabhängigkeit [7].

Regelmäßiger Tabakkonsum wird einerseits durch verhaltenswirksame operante und klassische Konditionierungsprozesse aufrechterhalten, wodurch die Mehrheit der Rauchenden eine ausgeprägte psychische Gewöhnung bildet. Zudem wirkt Nikotin in komplexer Weise auf dopaminerge, serotonerge, noradrenerge, glutamaterne und cholinerge Transmittersysteme und bewirkt eine dauerhafte Veränderung der Neurophysiologie und Neuroplastizität [8]. Vor diesem Hintergrund ist nachvollziehbar, dass es nur der Minderheit gelingt, den Tabakkonsum ohne Hilfe von außen zu beenden. Nur jeder fünfte Rauchende in Deutschland versucht, mit dem Rauchen aufzuhören [9]. Durch evidenzbasierte Tabakentwöhnungsinterventionen lässt sich der Abstinenzerfolg deutlich erhöhen.

\section{Tabakentwöhnung als adjuvante Therapie bei Tumorerkrankungen}

Tabakrauch ist auch als Risikofaktor für die Entwicklung von Bronchialkarzinomen und Kopf-Hals-Malignomen, v. a. dem Plattenepithelkarzinom, zu nennen, neben Alkoholmissbrauch und Infektionen mit dem humanen Papillomavirus. In einer internationalen Multicenterstudie des International Head and Neck Cancer Epidemiology Consortium konnte der Zusammenhang zwischen Tabakkonsum und der Entwicklung eines Kopf-Hals-Malignoms eindrücklich belegt werden [10]. Insbesondere Larynxkarzinome sind überproportional häufig mit Tabakrauchen assoziiert. Fortgesetzter Tabakkonsum im Rahmen sowie nach erfolgter Tumorbehandlung eines Kopf-Hals-Tumors erhöht außerdem das Risiko für ein Tumorrezidiv und die Ausbildung eines Zweitkarzinoms [11, 12]. Neben der negativen Beeinflussung des Überlebens zeigt sich ein kausaler Zusammenhang zwischen posttherapeutischer Fibrose und Tabakkonsum bei Patienten und
Patientinnen mit Kopf-Hals-Tumoren [13]. Eine verstärkte Fibrosierung beeinträchtigt relevant die Schluck- und Sprechfunktion sowie die Sensibilität im oberen Aerodigestivtrakt und hat somit direkten Einfluss auf das funktionelle posttherapeutische Ergebnis. Zusätzlich zeigt sich bei rauchenden Patienten und Patientinnen häufiger eine relevante Einschränkung der Zahn- und Mundgesundheit mit Parodontitis, Periimplantitis, Karies und Halitosis [14]. Rauchende mit postoperativer adjuvanter Radiotherapie zeigten außerdem ein deutlich erhöhtes Risiko für eine Osteoradionekrose [15]. Neben der Beeinträchtigung somatischer Organfunktionen durch den Tabakkonsum zeigen verschiedene Studien eine Interdependenz zwischen Substanzmissbrauch und negativen psychischen Symptomen wie Depressivität, Rezidivangst oder einer schlechteren Wiedereingliederungsrate in das Berufsleben bei Patienten und Patientinnen mit Kopf-Hals-Tumoren [16-18].

Aus diesem Wissen ergibt sich die dringende Notwendigkeit, Tumorpatienten bei der Rauchentwöhnung zu unterstützen. Eine erfolgreiche Tabakentwöhnung kann ein vergleichsweise günstiger zusätzlicher Baustein in der Behandlung von Tumorpatienten sein, um Folgeschäden zu vermeiden sowie Behandlungserfolge und die Lebensqualität der Betroffenen zu verbessern. Patienten und Patientinnen, die sich in stationäre Behandlung begeben müssen, befinden sich in einer vulnerablen Situation, in der sie oftmals sehr empfänglich für einen Rauchstopp sind. Mittlerweile hat daher die Empfehlung zur Rauchentwöhnung auch in den fachspezifischen S3Behandlungsleitlinien Einzug gehalten. So wird beispielsweise in der S3-Leitlinie „Diagnostik, Therapie und Nachsorge des Larynxkarzinoms" [19] die Empfehlung zum Tabakverzicht gegeben: „Die Adressaten dieser Leitlinie sollten ihre Patienten darauf hinweisen, den Tabakkonsum aufzugeben und den Alkoholkonsum weitgehend zu reduzieren“. Für die Behandlung von Patienten mit chronisch obstruktiver Lungenerkrankung (COPD) wurde eigens eine Leitlinie zur Tabakentwöhnung entwickelt [20]. Die kürzlich finalisierte deutsche S3-Leitlinie "Rauchen und Tabakabhängigkeit" [21] gibt Empfehlungen zum
Screening sowie zur Diagnostik und Behandlung von Tabakabhängigkeit.

Seit Mitte letzten Jahres wurde am Universitätsklinikum Leipzig (UKL) ein Tabakentwöhnungsangebot für Patienten und Patientinnen des Klinikums etabliert. Das „Rauchfrei-Programm”, das in der Ursprungsform vom Institut für Therapieforschung (IFT) entwickelt wurde, ergänzt die Behandlungsangebote des UKL und soll die Therapie verschiedener tabakassoziierter Erkrankungen der Lunge, des HerzKreislauf-Systems, aber auch von Tumorerkrankungen im Sinne eines ganzheitlichen Behandlungsansatzes unterstützen. Dabei werden Patienten und Patientinnen des Klinikums, die sich Unterstützung bei der Tabakentwöhnung wünschen, anhand einer Kurzberatung zu einem verhaltenstherapeutischen Gruppenprogramm („Rauchfrei-Programm“) oder anderen evidenzbasierten Tabakentwöhnungsmethoden vermittelt. Bei Bedarf wird das verhaltenstherapeutische Gruppenprogramm auch um Nikotinersatzpräparate oder Medikamente zur Dämpfung der Entzugssymptome ergänzt. Zudem schließt sich eine telefonische Weiterbetreuung zur Verhinderung von Rückfällen an.

\section{Diagnostik und Behandlung von schädlichem und abhängigem Tabakkonsum}

Die S3-Leitlinie "Rauchen und Tabakabhängigkeit" [21] empfiehlt in starkem Maße, dass bei allen Patienten und Patientinnen in allen medizinischen Fachrichtungen der Rauchstatus erfasst werden soll, unabhängig davon, ob eine tabakassoziierte Erkrankung vorliegt oder nicht. Neben den ICD-10-Kriterien zur Feststellung der Tabakabhängigkeit sind die gebräuchlichsten Instrumente zur Bestimmung des Schweregrades der Fagerström-Test für Zigarettenabhängigkeit [22] und der Heaviness of Smoking Index [23].

Nachdem der Rauchstatus erfasst wurde, soll das Behandlungsteam eine klare Empfehlung zum Rauchstopp geben. Eine Cochrane-Analyse [24] mit 31.000 Teilnehmern zeigte, dass ein Gespräch von Ärztinnen oder Ärzte mit Rauchenden über ihr Rauchverhalten im Rahmen einer Kurzberatung mit einer erhöhten Anmeldezahl zur Tabakentwöhnung verbunden ist. Das 
Gespräch kann effizient durch die Kurzberatung zur Tabakentwöhnung erfolgen, die dem „ABC-Schema“ in 3 Schritten folgt [25] und in einem konkreten Unterstützungsangebot endet. Das ABC-Schema beinhaltet die Schritte

- „Ask“: Abfragen und Dokumentation des Rauchstatus

- "Brief advice“: individuelle und motivierende Empfehlung zum Rauchstopp und

- "Cessation support": qualifizierte Unterstützung beim Aufhörwunsch oder Weiterleitung an bestehende anerkannte Entwöhnungsangebote (z.B. Telefonberatung, digitale Entwöhnungsangebote, Einzel- oder Gruppenschulung).

Durch offene Fragen wie: „Wie geht es Ihnen mit dem Rauchen?" oder "Was denken Sie selbst über Aufhören?" lässt sich eine Gesprächsatmosphäre schaffen, in der Betroffene häufig ein gewisses $M a ß$ an Aufhörbereitschaft signalisieren und dann konkrete Unterstützung angeboten werden kann. Eine empathische Gesprächsführung auf Augenhöhe vermeidet, dass sich die Betroffenen bevormundet fühlen und auf Abstand gehen. Umgekehrt kann ein nicht stattfindendes Ansprechen auch als ärztliches Einverständnis zum Rauchen verstanden werden.

Allen entwöhnungswilligen Rauchenden soll eine leitliniengerechte Behandlung angeboten werden [21]. Dabei wird sowohl eine psychotherapeutische als auch eine medikamentöse Unterstützung des Rauchstopps empfohlen. Zunächst sollen evidenzbasierte Angebote niederschwelliger Verfahren (Informationsbroschüren, Kurzberatung, Telefonberatung oder internet- bzw. App-gestützte Verfahren) gemacht werden. Mit einem umfassenden evidenzbasierten Angebot offeriert die Bundeszentrale für gesundheitliche Aufklärung (BZgA) Rauchenden und Exrauchenden vielfältige Unterstützungsmöglichkeiten wie individuelle Beratung über eine kostenlose Telefonhotline („Rauchfrei-Hotline“) oder Hilfe bei Rückfällen.

Kurzberatung, telefonisch oder persönlich, ist besonders dann wirkungsvoll, wenn sie proaktiv angeboten wird. Mit dem Angebot "rauchfrei ticket" der
BZgA hat jede Akut- oder Rehabilitationsklinik, egal welcher Fachrichtung, die Möglichkeit, den eigenen Patienten oder Patientinnen im Rahmen einer ärztlichen Minimalintervention ein Tabakentwöhnungsangebot zu unterbreiten, das sich in den Praxis- oder Klinikalltag integriert, indem die Betroffenen proaktiv durch das Beratungsteam der BZgA bis zu 6-mal telefonisch kontaktiert werden.

Gerade während der Coronapandemie sind digitale Ausstiegsangebote, durch die sich i.d. R. persönliche Kontakte vermeiden lassen, relevanter geworden. So können sich Aufhörwillige unter www. rauchfrei-info.de bei der BZgA für ein Onlineausstiegsprogramm anmelden. Das erste digitale, leitlinienbasierte Tabakentwöhnungsangebot, das zudem als digitale Gesundheitsanwendung zugelassen ist, d. h. bei Vorliegen der ICD-10-Diagnose F 17.2 ärztlich verordnet werden kann, ist "Nichtraucherhelden". Internetbasierte Programme sind wirksam [26] und eignen sich u.a. gut für medizinisch unterversorgte Regionen und Personen mit eingeschränkter Mobilität.

Bei Vorliegen eines ausgeprägten Schweregrads, körperlicher Abhängigkeit oder komorbiden Erkrankungen oder auf Wunsch des Patienten soll eine verhaltenstherapeutische Einzel- oder Gruppenbehandlung, ggf. auch in Verbindung mit einer Nikotinersatztherapie (Pflaster, Kaugummi, Inhalator, Mundspray oder Lutschtabletten) bzw. medikamentösen Therapie (zugelassene Wirkstoffe: Vareniclin, Bupropion oder (ytisin), vorgeschlagen werden. Zudem gibt es zahlreiche weitere Angebote wie Akupunktur, Hypnose, diverse internetgestützte Beratungsangebote und Apps, für deren Wirksamkeit es aber keine (eindeutige) Evidenz gibt. Das Deutsche Krebsforschungszentrum stellt umfangreiche Informationen zum Rauchen, zum Rauchstopp und zur Tabakkontrollpolitik zur Verfügung.

Die 12-Monats-Abstinenzraten professioneller Tabakentwöhnungsbehandlungen liegen je nach Interventionsform zwischen $25-40 \%[27,28]$. Anhand dieser Zahlen werden die löschungsresistenten Suchtmechanismen abhängig Rauchender deutlich.

\section{Gesundheitspolitische \\ Rahmenbedingungen für Tabakentwöhnung in Deutschland}

Obwohl es eine Vielzahl von Studien gibt, die die Wirksamkeit von Tabakentwöhnungsmaßnahmen belegen, wird evidenzbasierte Tabakentwöhnungstherapie bisher nur von einem kleinen Teil der Rauchenden in Anspruch genommen. Ein Grund ist, dass es bislang medizinischen Einrichtungen in Deutschland überwiegend selbst überlassen bleibt, welchen Stellenwert sie evidenzbasierten Interventionen zur Behandlung der Tabakabhängigkeit einräumen und ob und wie diese in Routineprozesse implementiert werden. Die Betroffenen informieren sich häufig selbst auf einem wenig regulierten Markt von Tabakentwöhnungsangeboten. Daher ist es dringend erforderlich, dass in Zukunft in allen Einrichtungen des Gesundheitswesens evidenzbasierte Interventionen zur Förderung des Rauchstopps und Algorithmen für die Zuweisung zu diesen Angeboten implementiert werden.

Maßgeblich mitverantwortlich für die zu geringe Inanspruchnahme von Tabakentwöhnungsangeboten ist auch die Tatsache, dass diese bisher nur von einigen Krankenkassen im Sinne einer Präventionsmaßnahme geringfügig bezuschusst werden, unter der Voraussetzung, dass noch keine tabakassoziierten Erkrankungen aufgetreten sind. Eine Pharmakotherapie (Nikotinersatztherapie, Medikamente) müssen Rauchende bisher vollständig selbst bezahlen. Eine Nutzung von Pharmakotherapie und evidenzbasierten Therapieangeboten ist jedoch wahrscheinlicher, wenn das Einkommen höher ist bzw. wenn die Kosten erstattet werden [9]. Mit der im Juni 2021 beschlossenen Übernahme von Arzneimittelkosten für den Rauchausstieg durch die gesetzliche Krankenversicherung haben Versicherte, bei denen eine schwere Tabakabhängigkeit festgestellt wurde, in Zukunft alle 3 Jahre Anspruch auf eine Übernahme dieser Kosten, wenn sie an einem evidenzbasierten Programm zur Rauchentwöhnung teilnehmen. Welche Medikamente und Therapiemaßnahmen unter welchen Voraussetzungen verordnet werden können, wird der Gemeinsame Bundesausschuss festlegen. Dies ist umso 
wichtiger, als der Anteil der Rauchenden, die versuchen, mit dem Rauchen aufzuhören, in den letzten Jahren gesunken ist [9] und das Einsparpotenzial der medizinischen Behandlungskosten in Deutschland durch einen Rauchstopp belegt wurde [29]. Zu hoffen ist, dass die Erstattung der Kosten durch die Krankenkassen einen erheblichen Beitrag für die häufigere Beratung durch Ärzte und Ärztinnen, für die Teilnahme an evidenzbasierten Tabakentwöhnungsmaßnahmen und für mehr Gesundheitsgerechtigkeit leisten wird.

Ärztinnen und Ärzte sollen nach den aktuell gültigen Leitlinien rauchende $\mathrm{Pa}$ tienten und Patientinnen professionell beraten und ihnen wirksame Entwöhnungstherapien anbieten, um rauchbedingte Erkrankungen zu verhindern oder den Verlauf bereits eingetretener Erkrankungen zu verlangsamen. Jedoch wird dies bisher im Medizinstudium kaum trainiert und viele Ärztinnen und Ärzte in Deutschland kommen dieser Aufgabe nicht adäquat nach [30], was ein weiterer Grund für die geringe Inanspruchnahme einer Tabakentwöhnungstherapie ist. Gleichzeitig herrscht ein hoher Grad an Unwissenheit hinsichtlich der Rauchentwöhnungsprogrammen sowohl bei Patienten und Patientinnen als auch bei Behandelnden [31], was sich mit den eigenen Erfahrungen der Autoren deckt. Maßnahmen zur Beratung und Behandlung von Rauchenden müssen deshalb stärker in die Aus- und Weiterbildung von Gesundheitsberufen integriert und Angehörige von Gesundheitsberufen in der Kurzberatung zur Förderung des Rauchstopps besser qualifiziert werden [21]. Die flächendeckende Umsetzung dieser Empfehlungen bedarf daher noch einer intensiven Schulung aller an der Behandlung von Menschen mit einer Krebserkrankung beteiligten Disziplinen. Eine kostendeckende Behandlung der Tabakabhängigkeit in Deutschland und ausreichende Steuerungsmechanismen dafür, dass dieses wichtige Versorgungsangebot in ausreichendem Umfang und ausreichender Qualität von professionellen Behandelnden umgesetzt werden kann, sind längst überfällig. Die Politik muss das Thema Rauchentwöhnung höher auf die Agenda setzen und bei den

Tobacco consumption and treatment of tobacco dependence. Effective strategies and current developments

Every year, around 127,000 people in Germany die as a result of smoking tobacco. These include 85,000 people with tobacco-related cancer. However, about a quarter of the adult population in Germany still smokes-often even when a tobaccorelated disease develops. Many smokers do not achieve abstinence without support. This article provides an overview of evidence-based tobacco cessation strategies and also describes the potential of tobacco cessation in the context of adjuvant therapy for tumor diseases. Finally, health policy challenges for tobacco cessation care are highlighted. A variety of psychological and pharmacological intervention methods for tobacco cessation are effective. Patients who smoke with tobacco-related diseases should be offered tobacco cessation therapy. As an adjuvant therapy in cancer treatment, it is comparatively inexpensive, with significant benefits for disease recovery and quality of life for those affected. To date, however, it has been offered in Germany only as a poorly regulated preventive measure. Tobacco cessation is of great importance as an adjuvant therapy for tumor diseases. In order to improve the quality of life of a large number of people with tobacco-related diseases, as well as for health economic reasons, it is urgently necessary that it become established and financed as a nationwide routine process in medical care.

\section{Keywords}

Nicotine $\cdot$ Smoking cessation $\cdot$ Cancer $\cdot$ Prevention $\cdot$ Intervention

behandelnden Ärzten und Ärztinnen zur Selbstverständlichkeit machen.

\section{Fazit für die Praxis}

- Tabakrauchen ist der größte vermeidbare Risikofaktor für chronische nichtübertragbare Krankheiten.

- Bei allen Patienten und Patientinnen soll der Rauchstatus erfasst werden und wirksame Tabakentwöhnungstherapien sollen flächendeckend routinemäßig entwöhnungswilligen Betroffenen angeboten werden.

- Tabakentwöhnung kann die adjuvante Therapie bei Tumorerkrankungen wirksam ergänzen.

- In Zukunft wird die Behandlung der Tabakabhängigkeit, auch bei Vorliegen tabakassoziierter Erkrankungen, in gewissem Umfang in den Leistungskatalog der Krankenkassen aufgenommen.

- Die Implementierung von evidenzbasierten Interventionen zur Behandlung des Tabakkonsums und Routineprozessen in die medizinische Versorgung muss durch weitere gesetzliche Vorgaben gesichert werden.

\section{Korrespondenzadresse}

Dr. rer. med. Dipl.-Psych. Katja Leuteritz Abteilung für Medizinische Psychologie und Medizinische Soziologie, Universitätsklinikum Leipzig

Leipzig, Deutschland

katja.leuteritz@medizin.uni-leipzig.de

Dr. rer. med. Dipl.-Psych. Katja Leuteritz Projektkoordination UKL Rauchfrei-Programm

\section{Einhaltung ethischer Richtlinien}

Interessenkonflikt. K. Leuteritz, V. Zebralla und H. Wirtzgeben an, dass kein Interessenkonflikt besteht.

Für diesen Beitrag wurden von den Autoren keine Studien an Menschen oder Tieren durchgeführt. Für die aufgeführten Studien gelten die jeweils dort angegebenen ethischen Richtlinien.

\section{Literatur}

1. https://www.dkfz.de/de/tabakkontrolle/ download/Publikationen/sonstVeroeffentlichun gen/Tabakatlas-Deutschland-2020_dp.pdf. Zugegriffen:21.Dez. 2021

2. https://www.dkfz.de/de/tabakkontrolle/ download/Publikationen/FzR/FzR_2019_ Rauchen-und-Lungenerkrankungen.pdf. Zugegriffen: 21. Dez. 2021

3. Clift AK, von Ende A, Tan PS, Sallis HM, Lindson N, Coupland CAC, Munafò MR, Aveyard P, HippisleyCox J, Hopewell JC (2021) Smoking and COVID19 outcomes: an observational and Mendelian randomisation study using the UK Biobank cohort. 
Thorax. https://doi.org/10.1136/thoraxjnl-2021217080

4. Chang CM, Corey CG, Rostron BL, Apelberg BJ (2015) Systematic review of cigar smoking and all cause and smoking related mortality. BMC Public Health 15:390

5. Inoue-Choi M, McNeel TS, Hartge P, Caporaso NE, Graubard BI, Freedman ND (2019) Non-daily cigarette smokers: mortality risks in the U.S. Am J Prev Med 56(1):27-37

6. https://www.bundesgesundheitsministerium.de/ service/publikationen/details/jahresbericht-derdrogenbeauftragten-2021.html. Zugegriffen: 21. Dez. 2021

7. Hughes JR, Helzer JE, Lindberg SA (2006) Prevalence of DSM/ICD-defined nicotine dependence. Drug Alcohol Depend 85(2):91-102

8. Schmidt HD, Rupprecht LE, Addy NA (2019) Neurobiological and neurophysiological mechanisms underlying nicotine seeking and smoking relapse Mol Neuropsychiatry 4(4):169-189

9. KotzD, BatraA, Kastaun S(2020) Smoking cessation attempts and common strategies employed. Dtsch Arztebl Int 117(1-2):7-13

10. Wyss A, Hashibe M, Chuang S-C et al (2013) Cigarette, cigar, and pipe smoking and the risk of head and neck cancers: pooled analysis in the International Head and Neck Cancer Epidemiology Consortium. Am JEpidemiol 178(5):679-690

11. Chen AM, Chen LM, Vaughan A, Sreeraman $R$, Farwell DG, Luu Q, Lau DH, Stuart K, Purdy JA, Vijayakumar S (2011) Tobacco smoking during radiation therapy for head-and-neck cancer is associated with unfavorable outcome. Int J Radiat Oncol Biol Phys 79(2):414-419

12. Cadoni G, Giraldi L, Petrelli L, Pandolfini M, Giuliani M, Paludetti G, Pastorino R, Leoncini E, Arzani D, Almadori G, Boccia S (2017) Prognostic factors in head and neck cancer: a 10-year retrospective analysis in a single-institution in Italy. Acta Otorhinolaryngol Ital 37(6):458-466

13. Pratson $\mathrm{CL}$, Larkins $\mathrm{MC}$, Karimian $\mathrm{BH}$, Curtis $\mathrm{CM}$, Lepera PA, Brodish BN, Ju AW (2021) The impact of smoking, alcohol use, recurrent disease, and Age on the development of neck fibrosis in head and neck cancer patients following radiation therapy. Front Oncol 11:707418

14. Ford PJ, Rich AM (2021) Tobacco use and oral health. Addiction 116(12):3531-3540

15. Treister NS, Brennan MT, Sollecito TP, Schmidt BL, Patton LL, Mitchell R, Haddad RI, Tishler RB, Lin A, ShadickR, Hodges JS, Lalla RV (2021) Exposed bone in patients with head and neck cancer treated with radiation therapy: An analysis of the Observational Study of Dental Outcomes in Head and Neck Cancer Patients (OraRad). Cancer 487-496. https:// doi.org/10.1002/cncr.33948

16. Balachandra S, Eary RL, Lee R, Wynings EM, Sher DJ, Sura T, Liu Y, Tillman BN, Sumer BD, Arnold EM, Tiro JA, Lee SC, Day AT (2022) Substance use and mental health burden in head and neck and other cancer survivors: a national health interview survey analysis. Cancer 128(1):112-121

17. van Liew JR, Christensen AJ, Howren MB, Hynds Karnell L, FunkGF (2014) Fear of recurrence impacts health-related quality of life and continued tobacco use in head and neck cancer survivors. Health Psychol 33(4):373-381

18. Broemer L, Friedrich M, Wichmann G, Müller J, Neumuth T, Dietz A, Mehnert A, Wiegand S, Zebralla $V$ (2021) Exploratory study of functional and psychological factors associated withemployment status in patients with head and neck cancer. Head Neck 43(4):1229-1241
19. https://www.awmf.org/uploads/tx_szleitlinien/ 017-076OLI_S3_Larynxkarzinom_2019-11.pdf. Zugegriffen:21.Dez.2021

20. https://www.dnrfk.de/fileadmin/user upload/website/dnrfk/OPS/Leitlinien_TE/ S3_Tabakentwoehnung_bei_COPD_2014-03. pdf.Zugegriffen:21.Dez. 2021

21. https://www.awmf.org/uploads/tx_szleitlinien/ 076-0061_S3_Rauchen-_TabakabhaengigkeitScreening-Diagnostik-Behandlung_2021-03.pdf. Zugegriffen:21.Dez. 2021

22. Heatherton TF, Kozlowski LT, Frecker RC, Fagerström KO (1991) The Fagerström test for nicotine dependence: a revision of the Fagerström tolerance questionnaire. Br J Addict 86(9):1119-1127

23. Heatherton TF, Kozlowski LT, Frecker RC, Rickert W Robinson J (1989) Measuring the heaviness of smoking: using self-reported time to the first cigarette of the day and number of cigarettes smoked per day. Br J Addict 84(7):791-799

24. Stead LF, Carroll AJ, Lancaster T (2017) Group behaviour therapy programmes for smoking cessation. Cochrane Database Syst Rev. https://doi. org/10.1002/14651858.CD001007.pub3

25. McRobbie H, Bullen C, Glover M, Whittaker R, Wallace-Bell M, Fraser T (2008) New Zealand smoking cessation guidelines. NZMed J 121(1276):57-70

26. Taylor GMJ, Dalili MN, Semwal M, Civljak M Sheikh A, Car J (2017) Internet-based interventions for smoking cessation. Cochrane Database Syst Rev. https://doi.org/10.1002/14651858. CD007078.pub5

27. Schepis TS, Rao U (2008) Smoking cessation for adolescents: a review of pharmacological and psychosocial treatments. Curr Drug Abuse Rev 1(2):142-155

28. Stead LF, Buitrago D, Preciado N, Sanchez G, Hartmann-Boyce J, Lancaster T (2013) Physician advice for smoking cessation. Cochrane Database Syst Rev. https://doi.org/10.1002/14651858. CD000165.pub4

29. Hering T, Batra A, Mühlig S, Nowak D, Rüther T, Schwarzkopf L (2021) Das Einsparpotenzial der Behandlungskosten bei COPD durch Rauchstopp - Modellierung für DMP COPD in Deutschland. Pneumologie 75(8):577-582

30. Twardella D, Brenner H (2005) Lack of training as a central barrier to the promotion of smoking cessation: a survey among general practitioners in Germany. Eur JPublic Health 15(2):140-145

31. Khodadadi $A B$, Carroll W, Lee EL, Hansen $B$, Scarinci IC (2021) It Takes Two to Tango: Patients' and Providers' Perspectives in Tobacco Cessation and Head/Neck Cancer. Oncologist 26(9):761-770

\section{German Cancer Survivors Week 2022}

30.05.-02.06.2022, Online

Wer an Krebs erkrankt, ist mit gesundheitlichen Einschränkungen konfrontiert, die sich auf die gesamte Lebenssituation auswirken: auf die Familie, den Freundeskreis und den Beruf. Die Deutsche Krebsstiftung möchte den Erfahrungsaustausch der Betroffenen fördern und zugleich Thema "Leben mit Krebs" in die Gesellschaft tragen. Deshalb veranstaltet sie seit 2015 den German Cancer Survivors Day: Betroffene und Expert*innen sprechen in der Öffentlichkeit über das Thema „Leben mit Krebs"

2021 wurde der German Cancer Survivors Day erstmals zur German Cancer

Survivors Week (GCSW). Auch in diesem Jahr findet die German Cancer Survivors Week statt, und zwar als gemeinsame Veranstaltung der Deutschen Krebsstiftung und den Landeskrebsgesellschaften Berlin, Hamburg, Nordrhein-Westfalen, Thüringen und Schleswig-Holstein. Der Bundesminister für Gesundheit Prof. Dr. Karl Lauterbach hat für die Veranstaltung die Schirmherrschaft übernommen.

Die Woche steht unter dem Motto „Niemand ist alleine krank". In der Zeit vom 30. Mai bis zum 02. Juni 2022 bietet das Programm einen informativen und interessanten Mix aus Online- und Präsenzformaten für Betroffene, ihre Angehörigen und Freund*innen. Mehr Details zur German Cancer Survivors Week 2022 finden Sie auf www.deutsche-krebsstiftung.de

\section{Kontakt:}

Iris Meumann

Deutsche Krebsstiftung c/o Deutsche Krebsgesellschaft +49 (0)30322932923 meumann@deutsche-krebsstiftung.de 
Hier steht eine Anzeige.

黑 Springer 